\title{
The Basic Problems of Contemporary Scientific View of the World
}

\author{
Joseph J. Smulsky \\ Institute of the Earth's Cryosphere, Siberian Branch of Russian Academy of Sciences, Tyumen, Russia \\ Email: jsmulsky@mail.ru
}

Received 1 July 2014; revised 20 August 2014; accepted 22 September 2014

Copyright (C) 2014 by author and OALib.

This work is licensed under the Creative Commons Attribution International License (CC BY).

http://creativecommons.org/licenses/by/4.0/

(c) (i) Open Access

\begin{abstract}
Contemporary scientific view of micro- and macro-world was built on a chain of successive hypotheses, each link of which has no reliable evidence. The examples of scientific concepts "Big Bang" and "neutrino" show the formation of erroneous hypotheses that lead to the emergence of these concepts. In addition, the accepted hypotheses lead to the emergence of new objects of scientific vision of the world, which in the real world are absent. The paper proves the fallacy of hypotheses about the dependence of mass on velocity, about particles of light, about light speed of gravity propagation, about "Black holes", about gravitational waves etc. This paper is the introduction to the book ${ }^{1}$, which, in turn, based on the book "The Electromagnetic and Gravitational Actions (The Non-Relativistic Tractates)". The last book was published 20 years ago and aroused great interest among readers. However, the developed methods in the book are not used in physics due to misconceptions about essence of science. The paper states a brief review of the book. This paper, as well as the book, will be useful to physicists, students, senior pupils and everyone who is interested in the scientific worldview.
\end{abstract}

\section{Keywords}

Erroneous Hypothesis, Big Bang, Neutrino, Mass Dependence on Velocity

Subject Areas: Functional Analysis, Fundamentals of Material Science

\section{Introduction}

Современная научная картина микро-и макромира является фальшивой [1]. Многие ее объекты являются воображаемыми и в действительности не существуют [2] [3]. С начала 20-ого века в науке утвердился метод описания окружающего мира на основании гипотез, которые выдвигаются с целью достижения

${ }^{1}$ Smulsky J.J. Electrodynamics of moving bodies. Determination of forces and calculation of movements. Saarbrucken, Germany: "Palmarium Academic Publishing”, 2014. 324 p. ISBN 978-3-659-98421-1. (In Russian) http://www.ikz.ru/ smulski/Papers/InfElMvBEn.pdf 
понимания мира. На основании гипотез создается теория, которая описывает и объясняет явление. Затем это вымышленное объяснение воспринимается за действительное устройство мира.

В этом заключается суть гипотезного метода описания и представления окружающего мира. В физических науках и в сопутствующих дисциплинах в настоящее время ему нет альтернативы. Некогда существовавшее ему оправдание: не подтвердившиеся гипотезы будут отбрасываться, а построенные на них теории будут забываться-сейчас не работает. Гипотезы, выдвинутые авторитетами, становятся господствующими. Еще говорят: становятся плодотворными. Добавим-высокорейтинговыми. А господствующие и высокорейтинговые положения всегда, во все времена подавляют то, что им противоречит. Поэтому при появлении новых фактов, противоречащих гипотезе, для их объяснения выдвигаются новые гипотезы. В итоге современная научная картина микро-и макромира построена на множестве последовательных предположений. С одной стороны она все дальше и дальше отодвигается от реальности, а с другой стороны, в силу противоречивости гипотез друг другу, в умах ученых, исповедующих их, происходит сумятица. Они не в состоянии выйти из этого воображаемого мира. Это с одной стороны. А с другой стороны, они его сами не понимают.

Приведу пример. Исследователь из Австралии, Стивен Крозерс, во многих своих работах [4]-[6] показывает, что в рамках общей теории относительности (ОТО) такие явления как “черные дыры”, расширяющаяся Вселенная и “Большой взрыв” не могут быть. Как известно, именно в рамках ОТО и были созданы гипотезы о существовании этих воображаемых объектов и развиты детальные их теории. Во многих своих статьях, выступлениях на конференциях и видеосюжетах С. Крозерс встречает понимание и одобрение. Однако, нет ни одного сторонника ОТО, который мог бы ему аргументировано возразить. Как я уже упомянул, не могут возразить по причине непонимания ОТО. Ситуация вопиющая! Нет ни одного ученого во всем мире, который понимает общую или специальную теорию относительности.

Приведу еще пример, как неподтвердившаяся гипотеза подкрепляется новой гипотезой. Из астрономических наблюдений стало известно, что у далеких объектов, галактик и квазаров, частота спектра излучения уменьшается. Говорят: происходит покраснение света. Явление уменьшения частоты звука или света источника известно, например, при его удалении. Называют его эффектом Доплера. В связи с этим была принята гипотеза о том, что далекие астрономические объекты удаляются от нас. Однако выяснилось, что уменьшается частота у таких объектов по всему небосводу. Тут бы отказаться от гипотезы. Нет, принимается новая гипотеза: происходит расширение Вселенной, вследствие чего все астрономические объекты, в том числе и мы с Солнечной системой, удаляются друг от друга.

Гипотеза расширения Вселенной противоречит здравому смыслу, если ее интерпретировать в прошлое. При этой гипотезе получается, что в прошлом все объекты Вселенной могли сблизиться на расстояния, меньшие их размеров. Это абсурд. Однако абсурда не будет, если принимать гипотезу о “Большом Взрыве”. Этой гипотезой можно объяснить сбор всех объектов Вселенной в одну точку, после которого происходит взрыв этой точки и расширение Вселенной. Сейчас говорят о плодотворности гипотезы “Большого взрыва”. Она породила целое направление в науке о зарождении Вселенной и о множестве процессов, которые создали материю во всех формах ее современного существования.

Дальнейшим анализом наблюдений выяснилось, что уменьшение частоты света происходит тем больше, чем дальше объект. Если объяснять это явление эффектом Доплера, то нужно принять, что более далекий объект удаляется быстрее от нас, чем более близкий. То есть разбегание объектов увеличивается с удалением их от центра расширения. Однако, это противоречит всему тому, что мы знаем. Все, что происходит на Земле и в наблюдаемой Вселенной, объясняется притяжением объектов друг к другу по закону тяготения Ньютона. В таком случае, если бы начался разлет объектов, скорость их с удалением от центра должна неизбежно уменьшаться. Почему? Потому что на каждый объект сила воздействия остальных направлена к центру совокупности, т.е. против скорости объекта. Это неизбежно приведет к уменьшению его скорости [3] [7] [8]. Эта ситуация идентична той, которую мы наблюдаем на Земле. Если камень бросить вверх, то с удалением его от поверхности Земли скорость камня будет уменьшаться, потому что сила земного тяготения направлена вниз.

Итак, факт о большем уменьшении частоты света у более дальних объектов по сравнению с более близкими противоречит первоначальной гипотезе, которая объясняла покраснение света эффектом Доплера. Казалось, логичным было бы отбросить эту гипотезу и все последующие гипотезы о расширении Вселенной и “Большом Взрыве”. Однако это не происходит. Чтобы оправдать увеличивающийся про- 
филь скоростей у расширяющейся Вселенной, вводят “темную энергию”. Ее назначение-создать силы отталкивания, которые будут преодолевать силы гравитационного притяжения между объектами, и заставить объекты ускоренно разбегаться от точки Взрыва. Теперь об этом достижении ученые, например, в научно-популярных американских фильмах или академик В.А. Рубаков, крупнейший специалист в теории ранней Вселенной, в своей видеолекции говорят примерно так. Ученые открыли “темную энергию”. Астрономы еще не обнаружили ее. Пока трудно представить, как ее можно обнаружить. Но она, несомненно, существует. Ибо без “темной энергии” не было бы расширяющейся Вселенной и “Большого взрыва”. Силы тяготения не позволили бы Вселенной расширяться все с увеличивающейся скоростью. А “темная энергия” создает силы отталкивания, которые позволяют это делать.

Таких примеров в современной научной картине мира, построенной на гипотезах, можно привести много. В предлагаемой читателю книге рассматриваются явления на основе безгипотезного подхода. Он заключается в изучении явления, измерении его свойств и установлении зависимостей между ними. Все это делается, чтобы понимать, при каких обстоятельствах явление происходит. Затем на основе полученных зависимостей создается его описание, которое можно назвать теорией явления. Она позволяет его предсказать или использовать для блага человека. В рассмотренном случае явление покраснения света у далеких астрономических объектов следовало бы также изучать. На Земле, утром и вечером, также происходит покраснение света. В этом случае солнечные лучи проходят наискосок через атмосферу, т.е. более длинный путь в атмосфере приводит к покраснению света. Не идентично ли это явление распространению света в межзвездной среде? Все это нужно исследовать. И требуется много времени: может даже не хватит жизни одного человека. А “плодотворную” гипотезу можно выдвинуть сразу и получить всемирное признание [9].

Выше я остановился на фальшивых объектах макромира. По сравнению с макромиром микромир меньше доступен восприятию человека. Его “увидеть” и то условно можно только с помощью специальных приборов и оборудования. Очень часто принципы работы этих устройств основаны на тех гипотезах, достоверность которых необходимо установить. Поэтому в этой области существует большая свобода для вымысла и фантазии.

В результате исследований электрических явлений в 18-19 веках были установлены силы взаимодействия между наэлектризованными и намагниченными телами. Например, если заряженные тела неподвижны, то силы притяжения или отталкивания между ними обратнопропорциональна квадрату расстояния между ними $R^{2}$. В конце 19 века возник вопрос, как изменится сила, если заряды будут двигаться. Здесь надо было бы изучать все обстоятельства. Однако ученые увлеклись гипотезами. Возобладала гипотеза А. Эйнштейна: если один заряд движется относительно другого со скоростью $v$, то сила остается такой же, но его масса увеличивается как $m / \sqrt{1-\beta^{2}}$, где $\beta=v / c$; с-скорость света. На этой гипотезе была построена Специальная теория относительности (СТО). Как любая гипотеза, это предположение противоречит множеству фактов. Когда записываются уравнения движения, нужно с таким же коэффициентом $\gamma=1 / \sqrt{1-\beta^{2}}$ изменять длину и время. Для сохранения первой гипотезы принимается следующая гипотеза: пространство и время при движении одного заряда относительно другого также изменяются с коэффициентом $\gamma$. А как быть при других, неэлектромагнитных взаимодействиях? Принимается следующая гипотеза: изменение массы, времени и пространства происходит при любых взаимодействиях относительно движущихся тел. Однако тело может двигаться относительно одного тела с одной скоростью, а относительно другого-с другой. Тогда как пространство, время и масса должны изменяться? Ответа на это противоречие нет в современной физике. Это не одно противоречие в СТО. Их очень много, и называют их парадоксами. Во множестве работ с критикой СТО парадоксам уделено достаточно внимания [10]-[19].

В предлагаемой читателю книге выполнено изучение электромагнитных взаимодействий. Выяснилось, что уже в первой половине 19 века были выполнены все измерения и на их основе установлены эмпирические Законы Кулона, индукции Фарадея и магнитного воздействия элемента тока Био-СавараЛапласа, которые позволяют определить силу воздействия движущегося заряда на неподвижный. Выяснилось, что эта сила зависит не только от расстояния между зарядами, но и от их скорости. Я нашел выражение для силы [20]. Из него следует, что при нулевой скорости $v=0$, сила определяется законом Кулона. С увеличением скорости величина силы уменьшается, и при скорости, равной скорости света, сила равна нулю. Таким образом, не масса, пространство и время изменяются с движением тела, а 
изменяется только сила его электромагнитного взаимодействия с другими телами.

Фальшивая гипотеза о зависимости массы от скорости привела к фальшивым построениям в микромире. Одним из таких построений является нейтрино [3]. Рекардо Церезани и Давид де Хилстер в своих работах [21]-[23] наглядно изложили ситуацию с нейтрино. Радиоактивный радий превращается в полоний и излучает электроны. В 1927 г. Ц.Д. Еллис и В.А. Вустер [24] с помощью калориметра измерили энергию электронов и получили ее величину $0.36 \mathrm{MeV}$. В 1931 г. Вольфганг Паули с позиции Теории относительности рассчитывает кинетическую энергию электрона и получает $1.16 \mathrm{MeV}$. Это значение обусловлено принятой в Теории относительности зависимостью массы электрона от скорости. В действительности масса неизменна, а сила зависит от скорости. Однако релятивисты считают, что такая ошибочная энергия $1.16 \mathrm{MeV}$ является реальной, поэтому придумывают частицу нейтрино, которая должна быть носителем фальшивой энергии $1.16 \mathrm{MeV}-0.36 \mathrm{MeV}=0.86 \mathrm{MeV}$.

Более полувека наукой ставятся грандиозные эксперименты по поиску нейтрино. Неизвестно, как его можно найти, т.к. нейтрино без взаимодействия может пройти насквозь хоть Землю, хоть Солнце. В разных радиоактивных превращениях нужно добавлять разные нейтрино с разными энергиями. Тем не менее, сейчас во все ядерные реакции введено нейтрино. Все энергетические их балансы экспериментально измерены. Экспериментальные величины теоретиками увеличены на фиктивную энергию нейтрино, и тут же они уменьшены на эту величину, за счет того, что все нейтрино беспрепятственно улетают.

Рассмотрение взаимодействий на основе реальных сил приведет к удалению из физики нереальных гипотез [8]. Рассмотрим еще несколько из них. Гипотеза о частицах света, так называемых фотонах. Свет и радиоволны являются электромагнитным воздействием одного тела на другое. Сила такого воздействия тела на заряженную частицу направлена перпендикулярно расстоянию ее от тела и колеблется по величине вокруг нулевого значения. Поэтому свет, как и радиоволны-не объект природы, не частицы. Свет-это свойство тел, которое заключается в воздействии одного тела на другое. Скорость движения тела и скорость распространения света-это не одно и то же. Свойства скорости распространения воздействия определяется вышеупомянутыми законами электромагнетизма. Они отличаются от свойств скорости движения тел. Этими отличиями обусловлено непонимание многих оптических опытов по распространению света между движущимися телами.

Гипотеза о световой скорости тяготения была введена для того, чтобы при гравитационном взаимодействии можно было бы использовать фальшивые гипотезы зависимости массы, длины и времени от скорости движения. Дополнительное обоснование эта гипотеза получила за счет того, что она якобы давала избыток вращения перигелия Меркурия, который не могла дать сила тяготения Ньютона. В действительности проблема с перигелием Меркурия не была достаточно исследована. Всесторонние исследования ее показали [7] [25]-[27], что световая скорость тяготения может дать прибавку вращения перигелия в 200 раз меньшую требуемой величины. Кроме того, уточненный избыток вращения перигелия в 1.5 раза больше. Далее, общая величина вращения перигелия по закону тяготения Ньютона рассчитывалась упрощенно: все тела рассматривались как материальные точки. Для самой близкой к Солнцу планеты, Меркурия, такое упрощение приводило к недостатку вращения перигелия. Учет не точечной формы Солнца объясняет все движения в Солнечной системе, в том числе и вращение перигелия [27]-[32].

Гипотеза о “черных дырах”. Чтобы преодолеть силу гравитационного воздействия Земли частице нужно сообщить вторую космическую скорость $v_{2 c}=11.2 \mathrm{\kappa м} / \mathrm{c}$, а для преодоления тяготения Солнца- $v_{2 c}=$ 500 км/с. Можно вообразить такую звезду, у которой $v_{2 c}$ равняется скорости света. Если принять гипотезу, что свет состоит из частиц, то такие частицы не смогут преодолеть тяготение звезды, и она для далекого наблюдателя представится темным телом, т.е. “черной дырой”. Это возможно, если силы тяготения действуют по закону Ньютона. В случае световой скорости распространения тяготения на движущуюся со скоростью света частицу воздействия не оказывается, и она от звезды, характеризуемой любой второй космической скоростью $v_{2 c}$, беспрепятственно улетит и достигнет наблюдателя.

Итак, гипотеза о “черных дырах" не имеет никаких оснований и со всех сторон ошибочна. Во-первых, свет-это не частицы. А, во-вторых, частицы света при световой скорости распространения тяготения свою скорость не изменяют.

Гипотеза об отклонении света. Согласно общей теории относительности (ОТО) луч света, проходящий вблизи звезды, например, Солнца должен искривиться. Это возможно в случае, если свет состоит из весомых частиц, и сила тяготения описывается законом Ньютона. При световой скорости тяготения, 
принятой в ОТО, воздействие звезды на движущуюся со скоростью света частицу не оказывается, и она движется прямолинейно без искривления своего пути. Итак, эта гипотеза ошибочна по тем же двум причинам, что и предыдущая.

Гипотеза о гравитационных волнах возникла в ОТО из теоретических предположений. Если гравитационное взаимодействие, как и электромагнитное, распространяется со скоростью света, то подобно электромагнитным волнам должны быть и гравитационные. Поэтому ошибочность гипотезы о световой скорости тяготения приводит к ошибочности гипотезы о гравитационных волнах.

Выше перечислены только главные гипотезы, на которых построена современная научная картина мира, не являются полным набором. Имеется множество второстепенных гипотез, а многие еще предстоит выявить. И все их нужно устранить. Кроме гипотез, в современном представлении о мире еще существует множество непонятных и противоречивых моментов, которые вызывают вопросы. Как правило, на эти моменты обращают внимание, когда встречаются с ними впервые. В дальнейшем они по-прежнему остаются неясными, но к ним человек привыкает и забывает об их существовании. Филипп Михайлович Канарев сформулировал более 2000 вопросов [14]-[16], которые необходимо выяснить в современных представлениях о мире.

Основу настоящей книги представляет вышедшая в 2004 г. моя книга “Электромагнитное и гравитационное воздействие (нерелятивистские трактаты)” [20]. Будучи молодым человеком, я задался целью изучить и разобраться во многих неясных вопросах, которые у меня возникали при знакомстве с теорией относительности. В процессе работы сформулировался безгипотезный подход к изучению окружающего мира. Для многих эта книга оказалась лучом света во мраке абсурдов Теории относительности. Приведу некоторые отклики на нее.

“Давно еще я говорил некоторым своим приятелям-физикам: займитесь и постройте электродинамику на основе принципа зависимости силы от скорости движения заряда... И вот теперь, наконец, вижу исполнение этой задачи... В правильности твоего пути я практически не сомневаюсь”.

23.01.95 г. Проф. В.В. Чешев, д.ф.н.: Давно еще я говорил некоторым своим приятелям-физикам: займитесь и постройте электродинамику на основе принципа зависимости силы от скорости движения заряда... И вот теперь, наконец, вижу исполнение этой задачи... В правильности твоего пути я практически не сомневаюсь".

25.02.95 г. Б.А. Бондарев, физик: ... Хочу отметить чрезвычайную простоту и ясность Вашей идеи, совершенно не встречающиеся теперь в научных публикациях... Впервые появилась настоящая научная работа по теории относительности и законам электромагнитного воздействия - это Ваша книга.

17.10.95 г. А.А. Долгов, физик: Вашу книгу читал до 3-00 ночи... Особенно понравилось объяснение неизменности массы, и полностью согласен, что взаимодействие двух заряженных частиц зависит от их относительной скорости.

1.03.96 г. Проф. В.В. Бугровский, д.т.н.: В молодости я много времени потратил, пытаясь найти неувязку у Эйнштейна, так как не мог принять выводов, следующих из его теории. Она казалась мне научной спекуляцией, каковой и продолжаю ее считать. К сожалению, мне не удалось найти таких неувязок, в результате чего я оставил занятия физикой и сосредоточил свои усилия на технике, в которой все держится на здравом ощущении реальности... Вам же это удалось. Ваши доводы не просто убедительны, они воодушевляют и дают надежду.

04.06.96 г. Prof. Oleg D. Jefimenko: ... То что “цензура” не пропускает работ, в которых гравитация исследуется без применения эйнштейновских гипотез, это чудовищное преступление перед наукой. Вам, фактически, пришлось заново открывать то, что когда-то было предложено Хевисайдом, ... хотя Вы идете в Вашей книге значительно дальше, чем Хевисайд.

G. Walton, SRL-1997.-V.1, N.3.-P.61: Проф. Смульский, несомненно, является наиболее убежденным и выдающимся оппонентом теории относительности; его статья важна для Special. Rel. Let.

Через несколько лет безгипотезный подход окончательно сформировался и его, а также решенные на его основе проблемы, я изложил в книге “Теория взаимодействия” [7]. В этой книге основные понятия я наполнил новым содержанием, кроме того не использовал понятий со старым содержанием и таких привычных понятий, как “поле”, “эфир” и др. Я считал, что исследователи смогут воспользоваться этой книгой, чтобы сразу включиться в работу по безгипотезному познанию мира. Однако сложившееся понимание целей и задач науки этому воспрепятствовало. Например, один ученый, доктор наук, прочитав эту книгу, сказал мне: “Тот путь, который Вы предлагаете, по-видимому, правильный, но я 
сомневаюсь, что по нему в ближайшие 300 лет кто-либо пойдет”.

В связи с этим я решил переиздать книгу [20], внеся в нее некоторые исправления и дополнения. Повидимому, она будет доступней исследователю, который стремится изучить все обстоятельства сложившейся картины мира и сам лично установить, что в ней фальшиво, а что-истинно.

В книге я решил изменить название, чтобы более точно отразить ее содержание. Настоящее название “Электродинамика движущихся тел. Определение сил и расчет движений” в полной мере ему соответствует.

После настоящего Предисловия идет Введение. Становление релятивистской электродинамики. В нем охарактеризованы представления начала 20 века, которые привели к появлению Теории относительности. Этот материал подготовлен великим философом из Томска, профессором Владиславом Васильевичем Чешевым. По моей просьбе он написал Предисловие к моей книге “Теория взаимодействия”. Эту книгу я задумал как руководство для исследователя, а в Предисловии, написанном философом, допускались различные позиции и трактовки. Поэтому я издал книгу без Предисловия В.В. Чешева. А в настоящей книге его Предисловие весьма уместно в виде настоящего Введения.

Как видно из приведенного выше отклика В.В. Чешева на мою книгу [20], у нас с ним одинаковое понимание в отношении описания электромагнитных взаимодействий. И если бы он не был философом, а был как я-механиком, то, возможно, он также пришел к тем же результатам, что и я. Вышедшая в 1984 г. книга В.В. Чешева “Проблема реальности в классической и современной физике” стала надежной опорой противников теории относительности. В Предисловии к своей книге [7] появление книги В.В. Чешева я назвал третьим этапом в становлении нерелятивисткого направления в науке. После своей книги В.В. Чешев опубликовал еще несколько работ о Принципе относительности. Все это свидетельствует, что представленный во Введении В.В. Чешевым анализ обстановки, выполнен в результате длительных исследований.

Настоящая книга состоит из отдельных статей, написанных в разные годы. Основной объем работ выполнен в 1968-1973 гг. Каждая статья строилась так, чтобы быть независимой от других. Но в первой главе "Безгипотезный подход к электродинамике и теории тяготения" представлена обобщающая работа, осуществленная в 1990 г. В ней систематизирован метод, показан логический путь его создания и приведены основные результаты. В последующих главах-со второй по шестую-даны обоснования положений первой главы.

Во второй главе проанализированы основания ТО, установлен смысл преобразований Лоренца. Показано, что принцип относительности и предельная скорость являются двумя ошибочными исходными положениями ТО.

В третьей главе в результате решения уравнений электродинамики получено выражение для силы взаимодействия двух точечных зарядов. Сила зависит не только от расстояния между ними, но и от их относительной скорости. Последнее свойство обусловлено тем, что электромагнитное воздействие распространяется с конечной скоростью.

Четвертая глава посвящена детальному анализу взаимодействия относительно движущихся наэлектризованных и намагниченных тел. Исследованы принципы описания взаимодействия двух неподвижных заряженных тел с помощью понятий массы и силы. Показано, что при правильном их определении связь между ними, выражающаяся вторым законом механики, не зависит от вида воздействия. Воздействие от движущегося заряженного тела выражается силой, которая зависит от скорости движения этого тела. Выведены силы воздействия заряженных точечного тела, пластины, отрезка, а также намагниченных тел и проводников с током на движущуюся относительно них заряженную частицу.

В пятой главе представлено логическое обоснование и дальнейшее развитие нового подхода описания взаимодействий быстродвижущихся тел. С его помощью рассмотрено движение частиц в ускорителях и показано, как их рассчитывать. Предложен способ ускорения частиц до сверхсветовых скоростей и выполнены необходимые расчеты. Технические возможности позволяют получить частицы со скоростью, превышающей в 1.3 раза скорость света. Многоступенчатые ускорители могут превысить и эту скорость. В приложении к главе 5 обсуждаются вопросы получения сверхсветовых частиц.

В шестой главе проанализирован выполненный в 1909 г. эксперимент Бухерера, который, как доказали К.Н. Шапошников и Н. Кастерин, противоречит ТО. Новый подход описывает результаты данного опыта. Здесь же в приложении, на основе классической механики, выведено соотношение для энергии вещества $E=m c^{2}$. 
В седьмой главе в результате численных решений уравнений движения получены траектории движения одного объекта относительно другого, взаимодействие между которыми распространяется со световой скоростью. Траектории, в отличие от классических, являются двухпараметрическими. При вариации определяющих параметров исследован весь спектр траекторий. Установлено, что с увеличением скорости угол между асимптотами гиперболовидных траекторий уменьшается и может даже стать отрицательным. При большом параметре взаимодействия скорость в перицентре достигает световой. Рассмотрены также параболо-и эллипсовидные траектории, отличие которых от классических заключается в повороте перицентра. Период конечных траекторий может быть в несколько раз меньше или больше $2 \pi$. Наблюдаются траектории “захвата” с орбиты одного радиуса на другой, а также со световой скоростью в перицентре. Показано влияние относительной скорости на гиперболовидные траектории при взаимодействии отталкивания. В результате асимптотических решений установлены в аналитическом виде границы между разными семействами траекторий. В предельных случаях траектории переходят в классические и релятивистские. Установлено, что релятивистское гравитационное взаимодействие более слабое по сравнению с классическим, поэтому “черные дыры” могут существовать при гравитационном воздействии, скорость которого значительно больше скорости света.

В Приложении представлены параметры и таблицы 92 траекторий движения тел при силе взаимодействия, зависящей от скорости. Здесь же для сравнения приведены параметры классических траекторий. Для удобства использования траектории систематизированы по разделам и подразделам, которые отражены в нумерациях траекторий.

Все замечания и пожелания просьба направлять по адресу: 625000 Тюмень, а/я 1230, Институт криосферы Земли СО РАН.

\section{References}

[1] Smulsky, J.J. (2007) Conceptual Error in Contemporary Science. Proceedings of the Natural Philosophy Alliance, 13th Annual Conference, University of Tulsa, OK, 3-7 April 2006, Vol. 3, Published Space Time Analyses, Ltd., Arlington, 277-281. http://www.ikz.ru/ smulski/Papers/CnErCS2.pdf

[2] Smulsky, J.J. (1996) The "Black Hole": Superstition of the 20-th Century. Apeiron, 3, 22-23. http://www.smul1.newmail.ru/English1/FounPhisics/BHAP2.doc.

[3] Smulsky, J.J. (2012) Letter to the Antirelativists. Proceedings of the Natural Philosophy Alliance, 19th Annual Conference, Vol. 9, Albuquerque, 25-28 July 2012, 567-568. http://www.worldsci.org/pdf/abstracts/abstracts_6667.pdf

[4] Crothers, S.J. (2008) Certain Conceptual Anomalies in Einstein’s Theory of Relativity. Progress in Physics, 1, 52-57.

[5] Crothers, S.J. (2010) The Kruskal-Szekeres “Extension”: Counter-Examples. Progress in Physics, 1, 3-7.

[6] Crothers, S.J. (2012) General Relativity-A Theory in Crisis. Global Journal of Science Frontier Research, Physics and Space Science, 12, 27-33.

[7] Смульский, И.И. (1999) Теория взаимодействия. Новосибирск: Из-во Новосиб. ун-та, НИЦ ОИГГМ СО РАН, 294 c. http://www.ikz.ru/ smulski/TVfulA5_2.pdf

[8] Smulsky, J.J. (2008) Real Forces and Unreal Hypotheses. Proceedings of the Natural Philosophy Alliance, 14th Annual Conference, University of Connecticut at Stors, USA, 21-25 May 2007, Vol. 4, Space Time Analyses, Ltd., Arlington, 240-241. http://www.ikz.ru/ smulski/Papers/RealFUH2.pdf

[9] Смульский, И.И. (2013) Анализ уроков развития астрономической теории палеоклимата. Вестник Российской Академии Наук, 83, 31-39.

[10] Xu, S.Z. and Xu, X.Q. (1994) A New Explanation of the "Mass-Velocity Relation”. Chinese Journal of System Engineering and Electronics, 5, 68-71.

[11] Renshaw, C. (1996) Apparent Superluminal Jets as a Test of Special Relativity. Apeiron, 3, 46-49.

[12] Jefimenko, O.D. (1997) Electromagnetic Retardation and Theory of Relativity. Electret Scientific Company, Star City, $306 \mathrm{p}$.

[13] Артеха, С.Н. (2007) Критика основ теории относительности. Издательство ЛКИ, Москва, 244 с.

[14] Kanarev, P.M. (2007) Questions on the Microcosm. Proceedings of the NPA, 14th Natural Philosophy Alliance Conference, Storrs, 4, 97-108.

[15] Канарёв, Ф.М. (2007) Начала физхимии микромира. 8-е издание. Краснодар, 750 с.

[16] Канарёв, Ф.М. (2013) Монография Микромира. Новые знания по фундаментальным наукам. Palmarium Academic Publishing, 588 c. 
[17] Li, Z.F., Li, T.J., Wang, C.J., Tian, X.M. and Wang, Z.Y. (2007) The Essence of Special Relativity and Its Influence on Science, Philosophy and Society. Proceedings of the Natural Philosophy Alliance, 13th Annual Conference, University of Tulsa, OK, 3-7 April 2006, Vol. 3, No. 1., 126-130.

[18] Li, Z.F. (2011) Observation Theory of Moving Objects. Physics Essays, 24, 34-38. http://dx.doi.org/10.4006/1.3533336

[19] Hajra, S. (2010) Some Experiments that Shook the World. Galilean Electrodynamics, 21, 13-17.

[20] Смульский, И.И. (1994) Электромагнитное и гравитационное воздействия (нерелятевистские трактаты). Наука, Новосибирск, 225 с.

[21] Carezani, R. (2011) Nuclear-Nuclear Collisions. http://www.worldsci.org/pdf/abstracts/abstracts_6242.pdf

[22] Carezani, R. (2011) Neutrinos at Fermi Lab. http://www.worldsci.org/pdf/abstracts/abstracts_6241.pdf.

[23] Hilster, D. (2011) The Neutrino: Doomed from Inception. Proceedings of the NPA, 8, 148-151. http://www.worldsci.org/pdf/abstracts/abstracts_6157.pdf

[24] Ellis, C.D. and Wooster, W.A. (1927) The Average Energy of Disintegration of Radium E. Proceedings of the Royal Society A, London, 117, 109-123.

[25] Смульский, И.И. (2008) Численное моделирование эволюции спутника вращающегося тела. В сб. Теоретические и прикладные задачи нелинейного анализа. Российская Академия Наук: ВЦ им. А.А. Дородницына. М.: ВЦ РАН А.А. Дородницына, 100-117. http://www.ikz.ru/ smulski/Papers/ModSun07c.pdf

[26] Smulsky, J.J. (2009) Gravitation, Field and Rotation of Mercury Perihelion. Proceedings of the Natural Philosophy Alliance, 15th Annual Conference, University of New Mexico, Albuquuerque, 7-11 April 2008, Vol. 5, No. 2, 254-260. http://www.ikz.ru/ smulski/Papers/08Smulsky2c.pdf

[27] Smulsky, J.J. (2011) New Components of the Mercury’s Perihelion Precession. Natural Science, 3, 268-274. http://www.scirp.org/journal/ns http://dx.doi.org/10.4236/ns.2011.34034

[28] Гребеников, Е.А. and Смульский, И.И. (2007) Эволюция орбиты Марса на интервале времени в сто миллионов лет. Сообщения по прикладной математике. Российская Академия Наук: ВЦ им. А.А. Дородницына. М.: ВЦ РАН А.А. Дородницына, 63 c. http://www.ikz.ru/ smulski/Papers/EvMa100m4t2.pdf

[29] Мельников, В.П. and Смульский, И.И. (2009) Астрономическая теория ледниковых периодов: Новые приближения. Решенные и нерешенные проблемы. Новосибирск: Академическое изд-во «Гео», 98 с. Книга на двух языках. С обратной стороны: Melnikov, V.P. and Smulsky, J.J. (2009) Astronomical Theory of Ice Ages: New Approximations. Solutions and Challenges. Academic Publishing House “GEO”, Novosibirsk, 84 p. http://www.ikz.ru/ smulski/Papers/AsThAnR.pdf

[30] Smulsky, J.J. (2012) Galactica Software for Solving Gravitational Interaction Problems. Applied Physics Research, 4, 110-123. http://dx.doi.org/10.5539/apr.v4n2p110

[31] Smulsky, J.J. and Smulsky, Y.J. (2012) Dynamic Problems of the Planets and Asteroids, and Their Discussion. International Journal of Astronomy and Astrophysics, 2, 129-155. http://www.scirp.org/journal/PaperDownload.aspx?paperID=23224 http://dx.doi.org/10.4236/ijaa.2012.23018

[32] Smulsky, J.J. (2012) The System of Free Access Galactica to Compute Interactions of N-Bodies. MECS, International Journal of Modern Education and Computer Science, 4, 1-20. http://www.mecs-press.org/ 